\title{
Los estilos tecnológicos cerámicos de La Chanchería como indicadores de modos de hacer y de producir de las sociedades tardías del valle de Uspallata (NO de Mendoza)
}

RMA

VI Jornadas

Arqueológicas Cuyanas
Pottery technological styles from La Chanchería as ways of doing and producing indicators from Uspallata Valley Late societies (Nortwestern Mendoza)

\author{
Vanina Victoria Terraza * y J. Roberto Bárcena** \\ *INCIHUSA-CONICET. E-mail: yepiana@hotmail.com \\ **INCIHUSA-CONICET/ FFyL-UNCuyo/UNLaR. E-mail: rbarcena@mendoza-conicet.gob.ar
}

\begin{abstract}
Resumen
Examinamos materiales cerámicos de componentes tardíos del área de La Chanchería (valle de Uspallata, NO de Mendoza), mediante el análisis morfométrico y decorativo. Seguimos criterios para la selección y obtención del Número Mínimo de Vasijas (NMV) y conformamos caracterizaciones para cada estilo cerámico, teniendo en cuenta morfología, capacidad volumétrica, composición general de las pastas y tipos de motivos decorativos.

Partimos de la idea de que cada estilo tecnológico es un modo de hacer, un conjunto de hábitos y capacidades apropiadas, en un tiempo y espacio determinados, y que involucra relaciones sociales cargadas de significado. La comparación de los resultados con el material arqueológico de otros sitios del área y de la región nos acerca a interpretaciones sobre comportamientos relacionados con la organización de la producción cerámica en tiempos del tardío local y de la dominación estatal incaica.
\end{abstract}

Palabras clave: análisis cerámicos; estilos tecnológicos; tardío local; dominación inca regional.

\begin{abstract}
We examined late ceramic components in the La Chanchería area (Uspallata Valley, Northwest Mendoza) through morphometric and decorative analysis. We followed criteria for selecting and obtaining the MNV (minimal number of vessels) and we shaped a characterization for each ceramic style, taking into account morphology, volume, material and decorative patterns. We believe that each technological style is a way of making, habits and appropriate skills in a certain time and place. We can also say that it has a social meaningful relationship. If we compare this archaeological material to others in the same area, we find that there are related behaviors with the organization of ceramic production in the late times and in state Inca domination times.
\end{abstract}

Keywords: Ceramic analysis; technological styles; local late times; regional Inca domination.

Nuestros estudios arqueológicos y etnohistóricos permiten sustentar hipótesis sobre los desarrollos de las sociedades del tardío local en el valle de Uspallata y área de montaña del NO de Mendoza, como asimismo sobre el papel que representaron en época de la dominación inca regional (Bárcena 2001, entre otros). Por lo tanto el tipo de relación que se establecería entre las poblaciones locales y los incas es pertinente a nuestra labor, que orientamos en esta oportunidad al estudio de la organización de la producción cerámica.

Nos centramos en los estudios realizados en La Chanchería (S32.57978, 069.35599), sector que en la actualidad está modificado por la construcción de un hotel y su área de servicios, la localización del barrio Villa Clarita, la roturación de campos (hoy abandonados) o bien remociones de suelos para establecer un área municipal de deportes, sumándose a la anterior modificación generada por la apertura de una pista para carreras de caballos.

Sin duda, el yacimiento fue más extenso de lo que hoy reconocemos como La Chanchería, habida cuenta de que ya en los años '40 del siglo XX, el propio C. Rusconi (1962) se refería a éste como Potrero La Chanchería, implicando una superficie libre de modificaciones antrópicas mayores que, podemos deducir, al menos se extendería por el sur hasta la ruta 7, por el norte hasta las proximidades de 
Las Bóvedas de Uspallata, alcanzando por el este el área del Arroyo Uspallata, y teniendo por límite occidental las estribaciones de los cerros sitos en esta dirección.

En la superficie de esta gran área modificada, se aprecian, todavía y con respecto a las etapas de la prehistoria regional (Bárcena 2001), concentraciones de material cerámico y lítico, mientras no se aprecian estructuras de planta. Por ende, nuestro registro cerámico proviene principalmente de la recolección superficial en los amplios límites del extenso yacimiento.

Sustentamos la hipótesis de que su posición intermedia, entre los sectores ocupados por las poblaciones locales del valle y los detentados por la expansión inca en el área (el conspicuo tambo de Ranchillos se halla a unos $10 \mathrm{~km}$ de distancia del probable sector nuclear de La Chanchería, que a su vez es relativamente periférico con respecto al que podría señalarse como sector de mayor ocupación prehistórica en el valle, vertebrado por el Arroyo Uspallata), podría haber implicado funciones de nexo, relacionándose a través de esa posición ambos espacios, con las implicancias socio-políticas y económicas, del contexto cultural de la época (Rusconi 1962; Schobinger 1971; Bárcena 1977, 1998b; Bárcena et al. 2015).

Podemos asimismo señalar que nuestras investigaciones indican que tal ubicación, que reputamos como estratégica en la relación inca-población local, es utilizada por grupos que preceden en el lugar los propios de la época de la expansión del Tawantinsuyu en el NO de Mendoza.

Con la secuencia que denotan nuestras excavaciones, debemos asimismo atender que la impronta inca, apreciada claramente en el valle longitudinal YalguarazUspallata con la infraestructura vial y de tambos, se desarrolla por el piedemonte alto, sin incidir hasta donde sabemos en otras ubicaciones de los núcleos de población preexistentes, excepto en el área de los arroyos Tambillos y San Alberto y en el área de La Chanchería, y de forma relativa, en sitios de fondo de valle como El Canal, Los Sauces, Las Bóvedas y Las Colonias.

Los estudios, que preceden a los nuestros en el área, son los de C. Rusconi (1962-III-), J. Schobinger (1971) y J. R. Bárcena (1977, 1998b, 2011, entre otros; Bárcena et al. 2015).

En nuestro caso, realizamos análisis tecnotipológico y morfoestilístico de las tecnologías cerámicas del sitio, estableciendo relaciones con los tipos alfareros propios de los sitios incaicos del valle de Uspallata que, en su momento, nos llevaron a postular que el sitio habría sido una avanzada incaica relacionada con el tambo de Ranchillos, asentada en áreas de poblaciones locales del valle (Bárcena 1998b; Bárcena et al. 2013, 2015; Terraza 2013).

\section{Lineamientos metodológicos y materiales}

El material arqueológico bajo estudio proviene mayoritariamente de contextos superficiales, registrado a partir de recolecciones. Los sondeos de excavación han arrojado, hasta el momento, escasa (aunque relevante) información. Nuestro abordaje se sustenta, entonces, en los lineamientos metodológicos de la arqueología superficial.

Trabajamos así con la noción de paisaje a escala de análisis local, el sitio arqueológico y su entorno socioambiental, como también desde una perspectiva regional, abarcadora e integradora. Concebimos al sitio como "un lugar con restos materiales de actividad humana discreto y potencialmente interpretable. Entendiendo por discreto, limitado con los bordes marcados al menos en cambios relativos en la densidad de restos" (Plog et al. 1976: 389).

En nuestro caso, consideramos sitios a aquellos sectores del paisaje con alta frecuencia de restos (sectores de 20 $\mathrm{m}$. de diámetro con más de 20 artefactos) y también a concentraciones (de menos de 20 objetos) y "hallazgos aislados" (non- site) (Binford 1992).

Nuestro interés también se centra en observar a los sitios desde una escala regional para identificar variabilidad en la densidad superficial de artefactos, así como en la distribución por área de variantes estilísticas específicas del material cerámico, por lo que decidimos delimitar nuestra área de estudio a una microrregión, definida como un área donde hay una continuidad espacio-temporal del comportamiento humano en el aprovechamiento y explotación de recursos (Ots 2005).

Nuestra microrregión comprende el Arroyo Uspallata por su margen occidental y sectores conexos, desde las cercanías a la locación de las Bóvedas hasta la actual ruta nacional 7 (Figura 1).

Utilizamos como estrategias metodológicas la prospección pedestre mediante sistema de transectas y de cobertura total, el registro de la posición de hallazgos y concentraciones mediante GPS (no hay presencia de estructuras arquitectónicas) y la excavación de sondeos en zonas de mayor concentración de material cerámico.

Para la recolección superficial, dividimos el área de trabajo en sectores, los cuales fueron subdivididos en zonas y parcelas más pequeñas. Combinamos una técnica de muestreo no probabilístico o dirigido de $50 \mathrm{~m}^{2}$ sobre las concentraciones cerámicas en cada sitio, con la propuesta de muestra selectiva de fragmentos cerámicos en base a rasgos diagnósticos (Feely y Ratto 2011) aplicada a todo el área de estudio.

Registramos trece concentraciones cerámicas/hallazgos aislados, los cuales fueron muestreados. Del conteo 


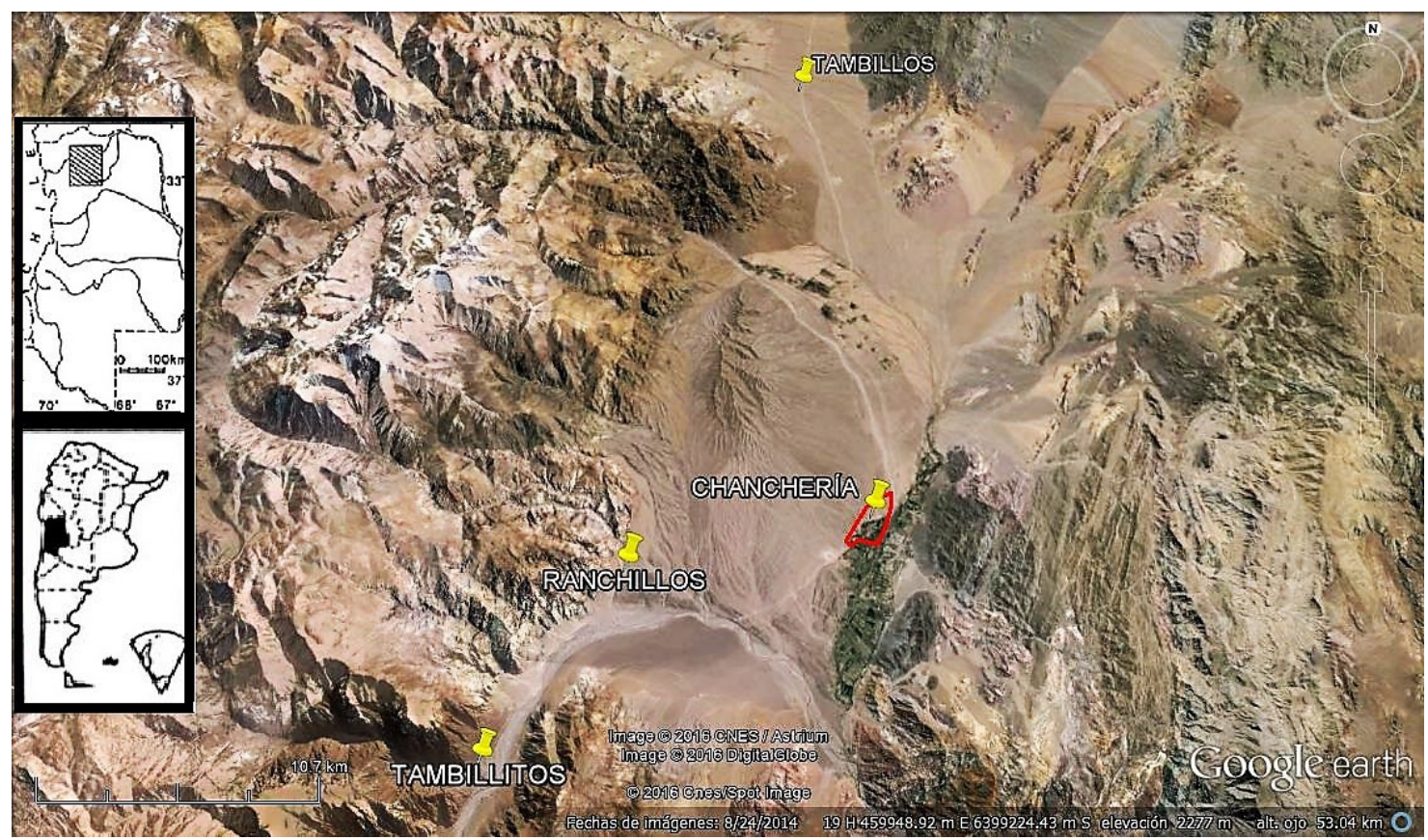

Figura 1: Mapa de la microrregión estudiada en el valle de Uspallata, con los sitios incas mencionados a lo largo del texto.

Figure 1: Map of the micro region showing the Inca sites, Uspallata valley.

\begin{tabular}{ccc}
\hline $50 \mathrm{~m} 2$ & Cantidad de fragmentos & total NMV \\
Concentración/hallazgo aislado & 3 & 3 \\
S1 O-Z4-5 & 7 & 7 \\
S1 O-Z9-4 & 3 & 1 \\
S1-O-Z14-1 & 2 & 1 \\
S1-O-Z16-4 & 8 & 6 \\
S1-O-Z16-5 & 3 & 3 \\
S1-O-Z16-7 & 19 & 14 \\
S1O-Z13-1(1) & 18 & 17 \\
S1O-Z13-1(2) & 23 & 20 \\
S1O-Z13-1(3) & 10 & 10 \\
S1O-Z13-2(4) & 3 & 1 \\
S1O-Z13-2(6) & 3 & 3 \\
S1O-Z13-2(7) & 102 & 86 \\
Total & &
\end{tabular}

Tabla 1: Resultados del cálculo de NMV (Número Mínimo de Vasijas) a partir del muestreo cerámico superficial en La Chanchería.

Table 1: MNV (Minimum Number of Vessels) results based on surface ceramic sampling from La Chanchería site.

resultó un total de 102 fragmentos, a los que se le aplicó el cálculo de número mínimo de vasijas (NMV) a partir de una agrupación cualitativa de fragmentos en función de distintos atributos morfotecno-estilísticos, combinándolos con un método cuantitativo de medición y conteo de partes diagnósticas (bordes, bases y cuerpos con puntos característicos) (Feely y Ratto 2011: 427). De esta operación se calcula un NMV de 86 vasijas (Tabla 1).

Realizamos catorce sondeos, de los cuales sólo cuatro presentaron material en estratigrafía (28 fragmentos en total). Los datos sobre los mismos no son presentados en este trabajo.

La distribución de las concentraciones cerámicas muestreadas se aprecia en la Figura 2.

Luego de haber realizado el cálculo del NMV, procedimos al análisis morfométrico mediante el remontaje, la fotografía de los tiestos y la reconstrucción de los perfiles respectivos. Con los softwares Rhinros y Matlab realizamos la reconstrucción 3D y calculamos la capacidad volumétrica de las vasijas.

A este procedimiento lo complementamos con la observación de atributos macroscópicos de los tiestos: color aparente y técnica de acabado de superficies, color y apariencia de las pastas. Observamos los fragmentos con lupa binocular, teniendo en cuenta la determinación de color (comparación con la paleta de la tabla Munsell -1994-) y presencia/ausencia de núcleo (a fin de asociar con la tecnología de cocción); identificación del tipo de fractura y textura de la pasta; identificación y descripción de inclusiones no plásticas (identificación de minerales y rocas, tiesto molido, etc.) y las cavidades o poros (para determinar tamaño y forma, abundancia y distribución); e identificación de huellas y alteraciones de uso y posdepositacionales (Cremonte, 1988; Orton et al., 1997; Cremonte y Bugliani 2010). El uso de la lupa binocular nos permitió clasificar de forma general las pastas y comparar con los tipos propuestos para el área.

Realizamos esta comparación teniendo en cuenta la revisión de la bibliografía correspondiente y el análisis 


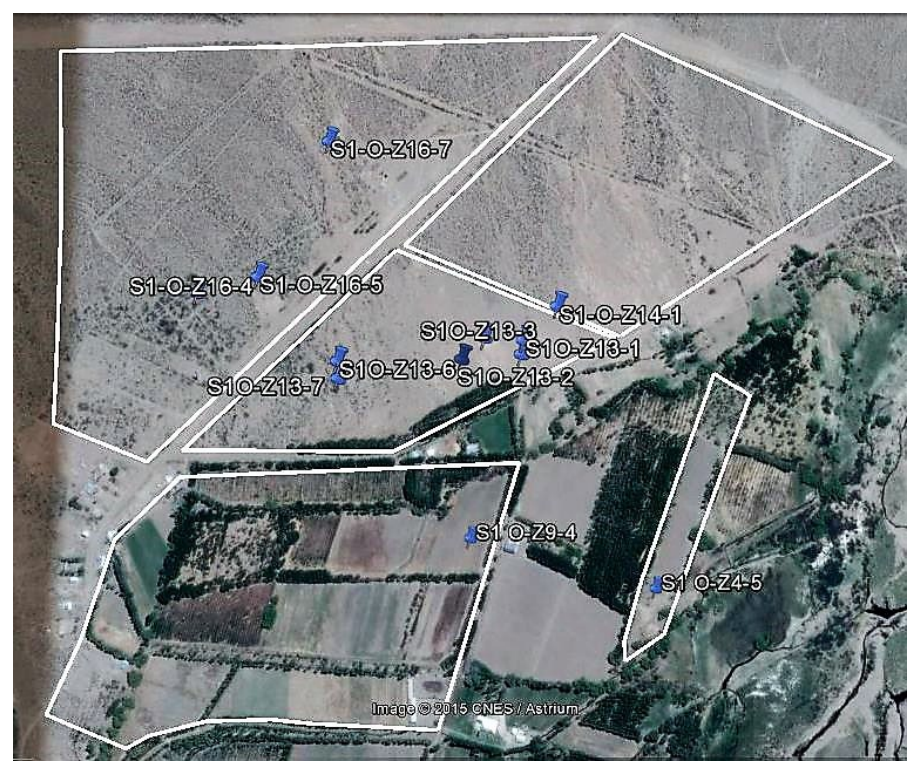

Figura 2: Distribución de las concentraciones cerámicas muestreadas en La Chanchería.

Figure 2: Ceramic distribution from La Chanchería site.

(que aún está en curso) de las colecciones museográficas de sitios tardíos e incas del valle de Uspallata.

\section{Los estilos tecnológicos de La Chanchería}

Entendemos a los estilos como agregados de recursos (plásticos, iconográficos, formales y técnicos) a los cuales apelan los grupos sociales locales, contribuyendo a conformar patrones de identidad compartidos y seguramente de imposición ideológica/política (Scattolin 2007). En consecuencia, la cerámica local, tradicionalmente asemejada con un "estilo inca", que ha sido, en algunos casos, identificada por los/las autores/ as que nos preceden como elementos de asignación incaica (Rusconi 1962; Durán y García 1989; Chiavazza 1995; Bárcena y Román 1990; Bárcena 1998b; García Llorca y Cahiza 1999; Cahiza 2001) y, en otros casos, denominada como Viluco o Viluco Inca (Lagiglia 1978, 2000; García 1994, 1996, 1999; Prieto O. 2005, 2007, 2010, 2012; Prieto O. y Durán 2007; Prieto O. y Chiavazza 2009), o bien como Inca Mixto (Raffino 1981; Ots 2007, 2008) es pensada no como un reflejo de influencias foráneas, o como índice de un proceso de modificaciones en las estructuras sociales a partir de la incorporación al Tawantinsuyu, sino como un agente activo de comunicación, subjetividad y representación de los conceptos que identificaron a los grupos integrados y dominados por el estado.

Del análisis morfométrico resultaron formas como ollas, cuencos o escudillas, platos y aribaloides. Estos últimos y los platos ornitomorfos responderían a relaciones con la presencia incaica. El cálculo de volúmenes y capacidades para estas formas se aprecian en la tabla 2.

Diferenciamos tres grupos generales de pastas que se corresponden con conjuntos estilísticos cerámicos bien determinados, en tanto que una parte de la muestra quedó sin definir en términos de estilos.

En La Chanchería reconocimos tres estilos, teniendo en cuenta el análisis morfométrico, macroscópico de pastas y decorativo (Figura 3):

Estilo A- Inca Mixto (en términos de Calderari y Williams 1991): registra fragmentos de pastas compactas, naranjas-rojizas, cocción oxidante. Pertenecen a vasijas de formas abiertas (como platos y pucos), con espesores que varían entre 4 y $6 \mathrm{~mm}$, y cerradas (aríbalos, aribaloides, ollas $u$ otros), con espesores de entre 6 a $9 \mathrm{~mm}$. En cuanto a las pastas, estas tienen una composición de partículas grises, blancas, negras, bordó, cuarzo y mica (y en algunos casos, calcita secundaria y/o tiesto molido), de esfericidad variable subredondeada-subangular y tamaño que varía entre fino y mediano, con distribución equilibrada y baja densidad. Algunas piezas se encuentran decoradas con pintura negra sobre engobes o pinturas rojas o marrones rojizas,

con diseños de bandas, reticulados oblicuos, romboides y rectángulos. Este estilo, de mayor distribución espacial (en sitios del sur de San Juan, norte y centro de Mendoza), pervive en la región hasta el siglo XVIII con transformaciones morfo-tecnológicas (Prieto O. 2010, 2012; Ots 2007, entre otros). Es interesante señalar que dentro de este conjunto se observan patrones decorativos que replican a los propiamente cuzqueños (como el reticulado oblicuo) o a los diaguitas (patrón ondas o espirales, bastones, escalonado, entre otros), pero con una manufactura local.

Estilo B-Diaguita (Fase III): representado por cerámicas de color marrón-rojiza, de pastas semicompactas y presencia de núcleo grisáceo, con o sin engobe crema, rojo/marrón, rojizo/violáceo, en sus superficies, generalmente pulidas, decoradas con pintura negra y/o roja en motivos como el reticulado, ajedrezado, rombos, cadenas, espirales, escalonado y bastones sobre la cara interna del fragmento (en casi la totalidad de los casos de formas abiertas), la cara externa o en ambas superficies. Las formas cerradas poseen un espesor entre 7 y $10 \mathrm{~mm}$, en tanto las abiertas de 4 y $6 \mathrm{~mm}$. Esta tradición alfarera acoge formas y diseños cuzqueños, pero conservando usualmente elementos diaguitas chilenos (Ampuero 1989, 1994; Cornely 1947, 1957). Sus pastas se componen por partículas blancas, negras, bordó, cuarzo y mica de esfericidad subangular y tamaño que varía entre fino y mediano, con distribución pobre y baja densidad.

Estilo C-Cerámica Gris: posiblemente de tradición Agrelo (de amplitud cronológica, que va del Período Temprano al Tardío regional) (Canals Frau y Semper 1956; Bárcena 1998c, 2001), de pastas porosas, espesor variable, algunas decoradas con incisiones o con modelados. La calidad de las terminaciones, de la textura y porosidad de 
las pastas es notablemente menor que en los primeros dos estilos. Las pastas están formadas por inclusiones negras, blancas, amarillas, cuarzo y mica, subangulosas, de tamaño fino, mediano y grueso, con distribución pobre y baja densidad.

Del cálculo del NMV, estos estilos quedaron representados de la siguiente manera: Estilo $A=52$, Estilo $B=6$, Estilo $C=16$, indeterminados $=12$. Total $N M V=86$.

De este muestreo datamos con TL dos fragmentos: uno de pasta naranja, decorado con pintura roja pulida en ambas caras (Estilo A) y otro de pasta gris con decoración incisa en su cara externa (Estilo C). El primero dio 1695 DC (320 \pm 30 años AP) y el segundo, 1485 DC (530 \pm 45 años AP). Estos fechados nos indican por un lado, la existencia de dos momentos (¿ocupaciones?) y por otro, la pervivencia del Estilo C durante el período tardío.

Mediante la revisión bibliográfica (Rusconi 1962, Schobinger 1971, Bárcena 1977, 1979, 1981, 1988, 1998 a y b; Bárcena y Román 1990; García Llorca 1995) y la indagación de la colección cerámica de Uspallata en el Museo Cornelio Moyano, pudimos observar que estos estilos cerámicos se hallan presentes en otros sitios del valle. El estilo A se encuentra en todos los sitios incas (Ciénaga de Yalguaraz, Tambillos, Tambillitos y Ranchillos) pero también en El Canal, Los Sauces, Las Bóvedas y Las Colonias. El estilo B está representado en Tambillos y Ranchillos, y además en Los Sauces y Las Colonias. El estilo C lo hallamos en los sitios de fondo del valle: El Canal, Los Sauces, Las Bóvedas y Los Álamos (cerrillo del Pucará).

\section{Conclusiones}

En sintonía con las publicaciones antecedentes sobre la temática (Prieto O. 2010, 2012; Prieto O. y Castro de Manchuca 2015; Prieto O. y Páez 2015; Prieto O. y Chiavazza 2015; Ots 2007; Bárcena et. al. 2015; entre otros), consideramos que las tradiciones tecnológicas locales se transformaron como consecuencia de la imposición incaica. Los cambios en los modos de hacer alfarerías se hacen patentes en lo tecno-morfológico -modificaciones en las composiciones de las pastas, adelgazamiento de paredes y creación de otras formascomo en la introducción de nuevos diseños decorativos.

Coincidiendo con los/las autores/as mencionados/as, sostenemos que la incorporación de nuevas tradiciones alfareras a la región dio origen a un estilo nuevo, nombrado por nosotros en este artículo como Estilo A, que tuvo amplia distribución espacial en el marco de las relaciones sociales, económicas, políticas y rituales de las poblaciones locales con los dominadores.

Esa distribución se da tanto a escala de la microrregión analizada como de la región del COA en general. Es interesante observar cómo la alfarería del estilo $C$ está representada en los sitios locales de fondo de valle,

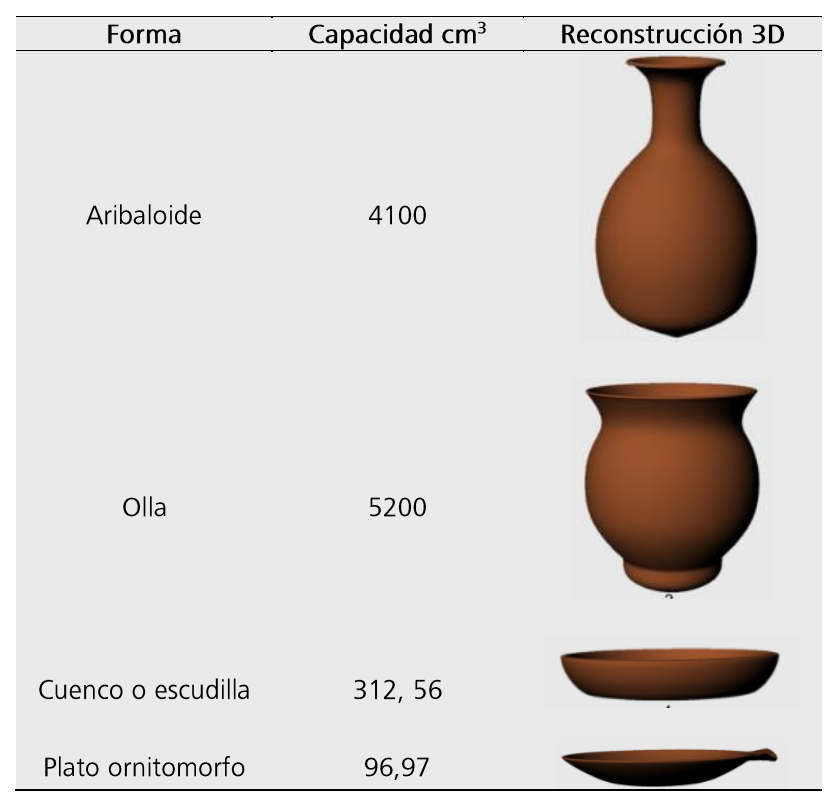

Tabla 2: Cálculo de la capacidad volumétrica y reconstrucción tridimensional de formas de La Chanchería.

Table 2: Volumetric capacity and three-dimensional forms reconstruction based on ceramic samples from La Chanchería.

cercanos a los cursos de agua (Arroyos Uspallata y San Alberto), en tanto que la de los estilos A y B la hallamos tanto en estos últimos como en los asentamientos incas ubicados a los pies de la Cordillera, alejados de las poblaciones locales de Uspallata y unidos por el Capacñam.

La transformación en los modos de hacer cerámica implicó asimismo nuevas relaciones de producción, pudiendo suponer el cambio desde una organización doméstica o comunal a otra centralizada, estandarizada, especializada, de acuerdo a las imposiciones de consumo estatal.

Tanto el Estilo A como el B están asociados con la expansión incaica en la región. La cerámica diaguita se halla en sectores de la cordillera (Sacchero y García 1991), del valle de Uco (Boman 1920), del valle de Uspallata (Bárcena 1998b; Bárcena y Román 1990; Rusconi 1962; Bárcena et al. 2015) de la precordillera (Durán y García 1989; García y Sacchero 1989) y de la planicie NE (Cahiza 1997, 2001). Para el valle de Uspallata, hay registro de cerámica con motivos incaicos y manufactura local en Tambillitos (Bárcena 1977), Chanchería (Rusconi 1962; Schobinger 1971; Bárcena et al. 2015), Los Sauces y El Canal (Rusconi 1962; Schobinger 1971); mientras que hay presencia de estilos foráneos como el Cuzco Policromo y el Inca Pacajes en Tambillos (Bárcena y Román 1990; Bárcena 1998b) o Inca Pacajes en San Miguel (planicie NE) (Chiavazza 2007).

Por lo tanto reafirmamos que en el Valle de Uspallata hay presencia de cerámica de producción foránea y 


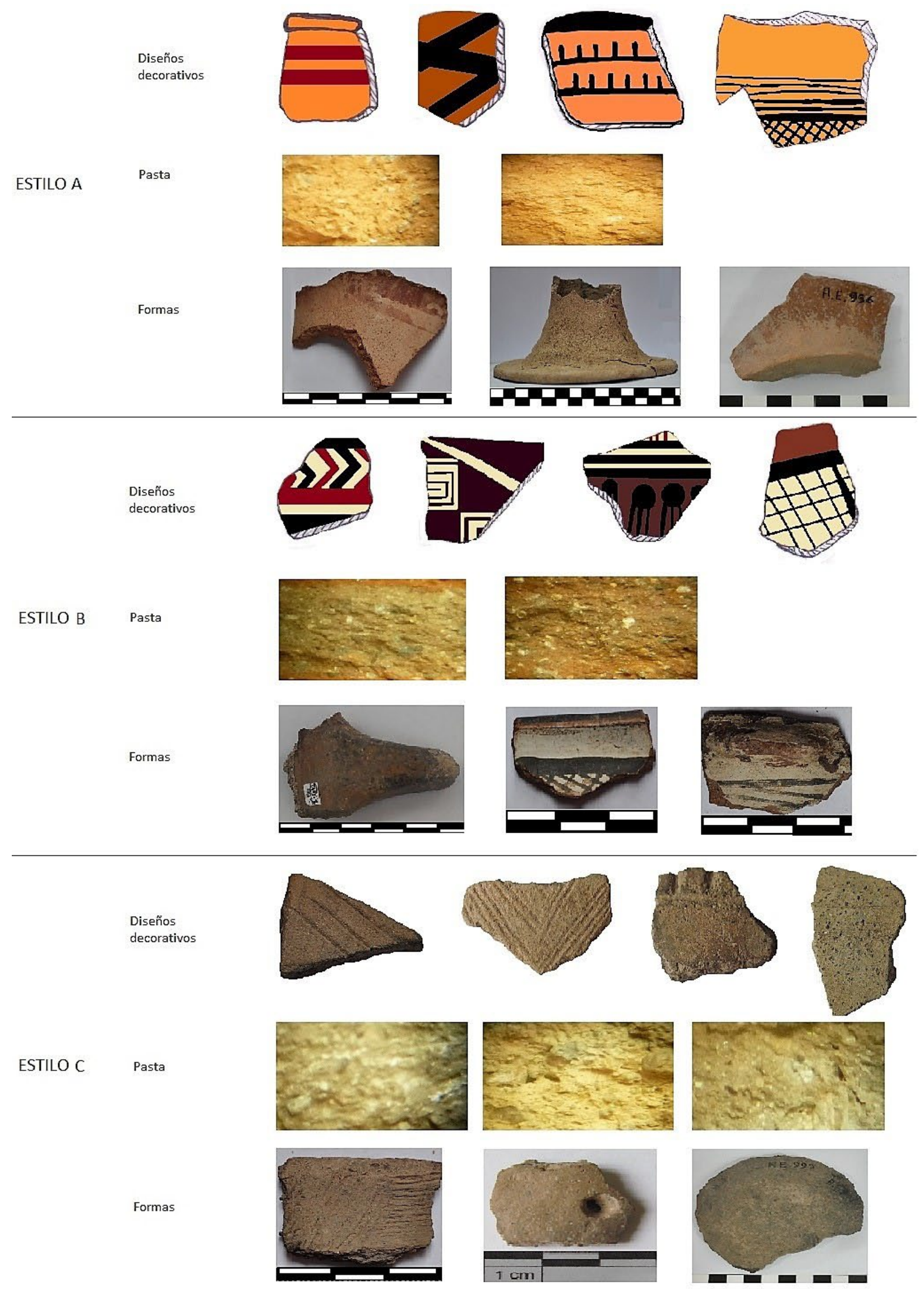

Figura 3: Los estilos tecnológicos de la Chanchería, teniendo en cuenta diseños decorativos, pastas y formas.

Figure 3: Technological styles from La Chancheria (includes decorative designs, pastes and shapes). 
local (Bárcena y Román 1990), y que la producción de cerámica local no sólo se transforma como resultado de los cambios estructurales impuestos o negociados que supone la dominación estatal, sino que lo hace además como respuesta comunicativa y subjetiva de los conceptos identitarios de las propias poblaciones locales dominadas.

Estas modificaciones en la organización de la producción cerámica se extendieron por toda la región del COA, tanto en sus valles centrales como en sectores periféricos a los mismos, como han considerado otros autores (Cahiza y Ots 2005), lo que nos hace pensar que la expansión estatal tuvo injerencia suficiente, concreta y con dinámica propia, en la frontera suroriental del Tawantinsuyu.

Mendoza, 20 de mayo de 2016.

\section{Bibliografía}

Ampuero G. 1989. La Cultura Diaguita Chilena. Prehistoria, J. Hidalgo et al. (Eds.), pp. 277-287. Editorial Andrés Bello, Santiago de Chile.

Ampuero G. 1994. Cultura Diaguita. Serie Patrimonio Cultural Chileno. Colección Culturas Aborígenes. División de Extensión Cultural del Ministerio de Educación, Santiago de Chile.

Bárcena, J. R. 1977. Informe sobre recientes investigaciones arqueológicas en el N.O. de la Provincia de Mendoza - Argentina (Valle de Uspallata y zonas vecinas) (Con especial referencia al período incaico). Actas del VII Congreso de Arqueología de Chile, vol. II: 661-692. Kultrún. Santiago de Chile.

Bárcena, J. R. 1979. Informe sobre recientes investigaciones arqueológicas en el N.O. de la provincia de Mendoza, Argentina (valle de Uspallata y zonas vecinas) (con especial referencia al período incaico). Actas del VII Congreso de Arqueología de Chile (Altos de Vilches, 1977). Tomo II: 661-692. Kultrún, Talca.

Bárcena, J. R. 1988. Investigación de la dominación incaica en Mendoza. El Tambo de Tambillos, la vialidad anexa y los altos cerros cercanos. Espacio, Tiempo y forma: Revista de la Facultad de Geografía e Historia, Serie I, prehistoria, Tomo I: 397-426.

Bárcena, J. R. 1998b. El Tambo Real de Ranchillos, Mendoza, Argentina. Xama, 6: 1-52, Publicación de la Unidad de Antropología, 11, Área de Ciencias Humanas, CRICYT, Mendoza.

Bárcena, J. R. 1998a. Arqueología de Mendoza. Las dataciones absolutas y sus alcances. EDIUNC. Mendoza.

Bárcena, J. R. 2001. Prehistoria del Centro Oeste Argentino. Separata de Historia Argentina Prehispánica. Editorial Brujas, Córdoba.

Bárcena, J. R. 2011. Arqueología en el Valle de Uspallata. Diario Los Andes, Sección A: 27. Mendoza.

Bárcena, J. R. \& Román, A. 1990. Funcionalidad diferencial de las estructuras del tambo de Tambillos: resultados de la excavación de los recintos 1 y 2 de la Unidad A del Sector III. Anales de Arqueología y Etnología t. XL-XLI: 7-81.

Bárcena, J. R., Terraza, V. V. \& Iniesta, M. L. 2015. Estudios tecnoestilísticos y de dataciones TL de materiales cerámicos del sitio La Chanchería (Valle de Uspallata, Noroeste de Mendoza,
Argentina). Arqueología y etnohistoria del Centro Oeste Argentino: Aportes desde las V Jornadas Arqueológicas Cuyanas. Monografías Xama 5. Zeta Editores. Mendoza.

Bárcena, J. R., Terraza, V. V., Sabatini G. I. \& Da Peña, G. V. 2013. Investigaciones arqueológicas recientes en el área del antiguo Potrero La Chanchería (Valle de Uspallata, Noroeste de Mendoza, Argentina). Actas del XVIII Congreso Nacional de Arqueología Argentina. Universidad Nacional de La Rioja.

Binford, L. R. 1992 Seeing the present and interpreting the past -and keeping things straight. En: Rossignol, J. y L. Wandsnider (eds.) Space, time and archaeological landscapes. Pp. 43-59. Plenum Press. New York-London.

Boman, E. 1920. Cementerio Indígena de Viluco (Mendoza) Posterior a la Conquista. Anales del Museo Nacional de Historia Natural 30: 501-562.

Cahiza, P. A. 1997. La presencia incaica en el noroeste de Mendoza: Análisis y experimentación cerámica. Seminario de Licenciatura (inédito). Facultad de Filosofía y Letras. Universidad Nacional de Cuyo, Mendoza.

Cahiza, P. A. 2001. Problemas y perspectivas en el estudio de la dominación inca en las tierras bajas de Mendoza y San Juan: el sitio Torre 285, Retamito. Xama. Mendoza. 173 - 197.

Cahiza, P.A. \& Ots, M.J. 2005. La presencia inca en el extremo sur-oriental del Kollasuyo. Investigaciones en las tierras bajas de San Juan y Mendoza y en el Valle de Uco -Rca. Argentina-. Xama, 15-18:217-228.

Caldelari, M. \& V. Williams. 1991. Re-evaluación de los estilos cerámicos incaicos en el noroeste argentino. Comechingonia. 9: 75-95.

Canals Frau, S. \& Semper, J. 1956. La cultura de Agrelo (Mendoza). Runa, VII (parte segunda): 169-187.

Cornely, F. 1947. Influencia incaica en la cerámica Diaguita chilena. Publicaciones del Museo y la Sociedad Arqueológica de La Serena N³: 10-13.

Cornely, F. 1957. Diaguita Chilena y Cultura El Molle. Editorial del Pacífico, Santiago.

Cremonte, M. B. 1988 (1983-1985) Alcances y objetivos de los estudios tecnológicos en la cerámica arqueológica. Anales de Arqueología y Etnología, 38-40: 179-217.

Cremonte, M. B. \& M. F. Bugliani, 2010. Pasta, forma e iconografía. Estrategias para el estudio de la cerámica arqueológica. Xama 19-23: 239-262.

Chiavazza, H. 1995. Estudios Arqueológicos en el sitio "Rincón de Los Helados" ("RH"). Ocupación Multicomponente en Noreste de Pampa de Canota - Departamento de Las Heras, Provincia de Mendoza, República Argentina. Tesis de Licenciatura, Facultad de Filosofía y Letras, Universidad Nacional de Cuyo, Mendoza. Inédita.

Chiavazza, H. 2007. Cambios ambientales y sistemas de asentamiento en el árido normendocino. Arqueología en los paleocauces del río Mendoza. Tesis para optar al título de Doctor en Ciencias Naturales. Facultad de Ciencias Naturales y Museo, Universidad Nacional de La Plata, La Plata. Inédita.

Durán, V. \& García, C. 1989. Ocupaciones agroalfareras en el sitio Agua de la Cueva Sector Norte (NO de Mendoza). Revista de Estudios Regionales 3:29-64. 
Feely, A. \& N. Ratto. 2013. Cálculo del número mínimo de vasijas y Recolección superficial: criterios metodológicos y análisis de casos del oeste tinogasteño (Catamarca). ANDES 24: 425-445.

García, A. 1994. Acerca de la Cultura material Durante el Período de Dominio Inca en Mendoza: dos casos de influencia Diaguita Chilena en la alfarería Viluco. Anales de Arqueología y Etnología 46/47: 41-48.

García, A. 1996. La dominación Inca en el centro oeste argentino y su relación con el origen y la cronología del registro arqueológico "Viluco". Anales de Arqueología y Etnología, 48/49: 41-48.

García, A. 1999. Alcances del dominio incaico en el extremo suroriental del Tawantinsuyu, Argentina. Chungará Revista de Antropología Chilena Vol. 29, № 2: 195-208.

García, A. \& Sacchero, P. 1989. Excavaciones arqueológicas en Agua de la Cueva- Sector Sur. Revista de Estudios Regionales 4: 27-51.

García Llorca, J. \& P. Cahiza. 1999. Análisis y experimentación cerámica: el índice de absorción como indicador tecnotipológico. Anales de Arqueología y Etnología 50/51: 23-38.

Lagiglia, H. 1978. La Cultura de Viluco del Centro Oeste Argentino. Revista del Museo de Historia Natural III (1-4): 227-265I.

Lagiglia, H. 2000. La Cultura de Viluco. Revista del Museo de Historia Natural de San Rafael, Mendoza. Manuscrito. Inédito.

Munsell Soil Color Chart. 1994. Macbeth Division of Kollmorgan Instruments Corporation. Revised Edition.

Orton, C., P. Tyers \& A. Vince. 1997. La cerámica en Arqueología. Barcelona, Crítica.

Ots, M. J. 2005. Prospección arqueológica de la cuenca del río de Las Tunas (departamentos de Tupungato-Tunuyán, Mendoza). Cuadernos de la Facultad de Humanidades y Ciencias SocialesUniversidad Nacional de Jujuy, 29: 43-60.

Ots, M. J. 2007. La presencia incaica en el Valle de Uco, Mendoza. Tesis doctoral inédita. Universidad Nacional de Cuyo.

Ots, M. J. 2008. Estudio de alteraciones provocadas por laboreo agrícola sobre conjuntos cerámicos en el sitio arqueológico Agua Amarga (Tupungato, Mendoza). Chungará. Revista de Antropología Chilena, 40: 145-160.

Plog, S., Plog, F., \& W. Wait. 1976. Decision making y modern surveys. Advances in Archaeological Method and Theory 1. Ed. por M. Shiffer, Academic Press, 384-421. New York.

Prieto Olavarría, C. 2005. Alfarería Viluco en el norte y centro de la Provincia de Mendoza (Argentina): nuevas perspectivas analíticas. Memoria para optar al título de Arqueólogo. Facultad de Ciencias Sociales, Universidad de Chile, Santiago. Inédita.

Prieto Olavarría, C. 2007. Cambios en la producción cerámica Viluco en los siglos XVI y XVII. Norte y Centro de Mendoza.
Actas XVI Congreso Nacional de Arqueología Argentina II: 3897395, Jujuy.

Prieto Olavarría, C. 2010. La especialización artesanal alfarera de la cultura Viluco. Norte y centro de la provincia de Mendoza. Tesis doctoral inédita. Facultad de Filosofía y Humanidades, Universidad Nacional de Córdoba, Argentina.

Prieto Olavarría, C. 2012. Perspectivas sobre el cambio tecnológico entre los períodos Medio y Tardío en el Norte de Mendoza. Aportes desde los estudios petrográficos. Resúmenes de Ponencias V Jornadas Arqueológicas Cuyanas, 31, Mendoza.

Prieto Olavarría, C. \& B. Castro de Manchuca. 2015. Petrografía, reconocimiento de fuentes de materias primas cerámicas y distancia a los recursos. El caso de la cerámica Viluco del norte de Mendoza. VI Jornadas Arqueológicas Cuyanas. San Rafael

Prieto Olavarría, C. \& H. Chiavazza. 2009. La producción cerámica Viluco entre los siglos XV y XVII (Provincia de Mendoza, Argentina). Chungará 41, 2, 261-274.

Prieto Olavarría, C. \& H. Chiavazza. 2015. Cambios en contextos de colonización: opciones económicas y transformaciones tecnológicas en el norte de Mendoza entre los siglos XV y XVII (Rca. Argentina). Vegueta. Anuario de la Facultad de Geografía e Historia 15: 159-184.

Prieto Olavarría, C. \& V. Durán. 2007. Cementerio indígena de Capiz Alto (San Carlos Mendoza). Actas del XIV Congreso Nacional de Arqueología Argentina Tomo 1: 225-238. Facultad de Humanidades, Universidad Nacional de Rosario, Rosario.

Prieto Olavarría, C. \& C. Páez. 2015. Presencia de inclusiones piroclásticas en la cerámica de los siglos XV a XVII en el Centro Oeste y Noroeste Argentino. Chungara, Revista de Antropología Chilena. 47, (3): 441-453.

Raffino, R. 1981. Los Inkas del Kollasuyu. Origen naturaleza y transfiguraciones de la ocupación Inka en los Andes Meridionales. Ramos Americana Editora, Buenos Aires.

Rusconi, C. 1962. Poblaciones pre y poshispánicas de Mendoza. III, Arqueología. Mendoza.

Sacchero, P. \& García, A. 1991. Una estación trasandina Diaguita Chilena. Actas del XI Congreso Nacional de Arqueología Chilena Tomo III: 61-67. Santiago.

Scattolin, M.C. 2007. Estilos como recursos en el Noroeste Argentino. María Clara Rivolta et al; dirigido por Axel E. Nielsen (Ed), Procesos sociales prehispánicos en el sur andino: la vivienda, la comunidad y el territorio, 291-321. Brujas, Córdoba.

Schobinger, J. 1971. Arqueología del Valle de Uspallata. Provincia de Mendoza (sinopsis preliminar). Relaciones 5, N.S., 2: 71-84.

Terraza, V. 2013. Desarrollos locales y dominación inkaica en el Valle de Uspallata (N.O. de la Prov. de Mendoza): Aportes a los estudios ceramológicos del sitio Potrero La Chanchería I. Tesis de grado. Facultad de Filosofía y Letras, Universidad Nacional de Cuyo, Argentina. 